\title{
Testing positive pressure delivered from commercial and WHO-style pediatric bubble CPAP devices
}

\author{
Nicholas A. Ettinger ${ }^{1 *}$, Nathan Serazin ${ }^{1}$, Richard Nguyen², Jennifer Werdenberg ${ }^{3,4}$, Minke Huibers ${ }^{5,6}$ and \\ Susan Torrey ${ }^{7}$
}

\begin{abstract}
Background/aim: Low-cost commercial bCPAP devices have been deployed in resource-limited settings to treat neonatal respiratory failure. The use of these devices has increased access to pediatric respiratory support for infants. However, constrained resources may result in substitution of recommended consumables and/or use in older age groups. We hypothesized that commercially available bCPAP devices, the standard WHO-style device and various improvised adaptations would all generate effective, safe positive pressure at the patient interface.
\end{abstract}

Methods: Performance of 2 commercially available bCPAP devices was tested against the standard WHO-style bCPAP device, as well as several improvised modifications of these devices, by measuring positive pressure delivered at the patient interface. Variables tested included different flow rates, patient interfaces and respiratory circuit tubing.

Results: Both commercial devices utilized according to manufacturer recommendations generated the expected positive pressure at the patient interface. When testing the recommended WHO-style bCPAP device with recommended materials as well as other improvised modifications, we found variable and potentially unpredictable generation of positive pressure at the patient interface.

Conclusions: Modified or improvised bCPAP devices should be used with extreme caution as the support provided may be more or less than expected depending on respiratory tubing and flow rates employed. Our data support the effectiveness of bCPAP in newborns and young infants. But, to our knowledge, there are no bCPAP patient interfaces for older children effective with low liter flow devices. Therefore, based on these results, we recommend against using WHO-style bCPAP devices for non-infant patients with respiratory failure and instead recommend using standard oxygen therapy with nasal cannulae or face-masks, as well as early consideration of transfer to a higher level of care.

Keywords: Pediatrics, Bubble CPAP, Critical care, Global health, Resource limited setting; respiratory failure

\section{Background}

Respiratory diseases are the most common cause of morbidity and mortality in children under 5 worldwide [1]. In resource limited settings (RLS), options for respiratory

\footnotetext{
*Correspondence: nicholas.ettinger@bcm.edu

1 Division of Critical Care, Department of Pediatrics, Baylor College of Medicine/Texas Children's Hospital, 6651 Main Street, MC: E1420, Houston, TX 77030, USA

Full list of author information is available at the end of the article
}

support are often constrained. Many locations lack both expertise and access to invasive respiratory support and/ or basic pediatric intensive care [2]. Bubble Continuous Positive Airway Pressure (bCPAP) has been proposed as an alternative to meet the need for respiratory support for infants and small children for which several commercial, durable, low-cost devices are available (e.g., Pumani) [3-6]. Studies have demonstrated that bCPAP can be effective, safe and can reduce morbidity/mortality 
in infant populations [3]. However evidence is mixed regarding the efficacy of bCPAP to improve outcomes in older children with respiratory failure in RLS [7, 8].

In addition to commercially available devices, instructions on how to create "low-tech" modified bCPAP devices with readily available resources have been published, [9] endorsed by the WHO (Figs. 16 \& 17 [10]) and promulgated in pediatric global health training websites [11]. In practice, these devices may be subject to large variability in construction based on the availability of specific equipment. This may variably affect the amount of actual positive pressure delivered. Oxygen source, delivered liter flow rates (liters per minute, lpm), circuit tubing, nasal or facial interface, miscellaneous connections, and the reliability of the bubble CPAP chamber are several of the patient-independent variables subject to potential local modification. The efficacy of positive pressure generation at the patient interface by variations of WHO-style devices has been incompletely studied [12]. The aim of our study was to measure the amount of positive pressure generated by commercially available bCPAP devices, the standard WHO-style device and various improvised adaptations. We hypothesized that all would generate effective positive pressure (PP) matching the submersion depth at the patient interface.

\section{Materials and methods}

\section{Control devices}

To test our hypothesis, we used the Fisher \& Paykel (F\&P) Healthcare Bubble CPAP with Flexitrunk ${ }^{\mathrm{TM}}$ and Pumani Bubble CPAP devices as controls. The F\&P device was chosen as a representative device used in high-resource settings. It requires a standard high-flow/high-pressure air/oxygen wall interface for air flow along with a proprietary humidifier chamber and self-contained pressure release valve (valve closed for all F\&P device testing). The Pumani bCPAP apparatus is a low-cost, durable, Conformitè Europëenne-approved commercial apparatus specifically designed for RLS $[4,5]$. It has a self-contained electric flow pump for air flow. Each was assembled with the manufacturer provided components (respiratory tubing, connections, humidifiers, patient interfaces, etc.) according to the manufacturer's specifications. Both were tested at air-flows recommended by the manufacturers (8lpm for the F\&P device and 6-8 lpm for the Pumani).

\section{Patient interfaces tested for the control devices (Table 1,}

Fig. 1)

For the F\&P device, we tested F\&P nasal prongs (sizes 3020, 3520, 4030, 4520, 5050 and 6570, Fig. 2) and F\&P nasal masks (sizes small, medium, large, extra-large, Fig. 3). For the Pumani device, we tested two of the standard Pumani nasal prongs (Hudson, sizes 0[small], 5[large], Fig. 4).

\section{Test devices}

The test devices (Table 1, Fig. 1) included the (\#1) WHOstyle recommended bCPAP device [9] and (\#2) improvised modifications to the WHO-style device, (\#3) the F\&P device with modifications and (\#4) the Pumani device with modifications. Our choice of improvised modifications was informed by observations in RLS at the bedside by our author group.

For the WHO-style recommended bCPAP device (\#1), we followed instructions as outlined in several commonly referenced global health sources, $[9,10]$ using a standard

Table 1 Configurations and Variables Tested - commercially available devices and improvised modifications

\begin{tabular}{|c|c|c|c|c|}
\hline Variation & Air Flow Source & bCPAP Chamber & Tested Device Tubing & Patient Interfaces Tested \\
\hline \multicolumn{5}{|c|}{ Commercially Available Devices } \\
\hline Fisher \& Paykel (F\&P) & $\begin{array}{l}\text { Standard high-flow/high pres- } \\
\text { sure air/oxygen wall interface }\end{array}$ & $\begin{array}{l}\text { F\&P bubble CPAP chamber } \\
\text { (F\&P\# BC100) }\end{array}$ & F\&P bCPAP tubing & $\begin{array}{l}\text { - F\&P Nasal Prongs } \\
\text { - F\&P Nasal Masks } \\
\text { - Standard Resuscitation Mask }\end{array}$ \\
\hline Pumani & $\begin{array}{l}\text { Internal air flow pump }+/- \text { sup- } \\
\text { plemental low-flow } \mathrm{O}_{2}\end{array}$ & Pumani bubble CPAP chamber & $\begin{array}{l}\text { - Standard Pumani } \\
\text { tubing } \\
\text { - Standard Pumani tub- } \\
\text { ing + F\&P Flexitrunk }{ }^{\mathrm{TM}} \\
\text { connector }\end{array}$ & $\begin{array}{l}\text { - Pumani Nasal Prongs } \\
\text { - F\&P Nasal Masks } \\
\text { - Sleepweaver }{ }^{\mathrm{TM}} \text { BIPAP mask }\end{array}$ \\
\hline \multicolumn{5}{|l|}{ WHO-Style Devices } \\
\hline WHO & $\begin{array}{l}\text { Standard low-flow wall oxygen } \\
\text { flow-meter (1-10 lpm) }\end{array}$ & F\&P bubble CPAP chamber & $\begin{array}{l}\text { Pediatric \& Adult size } \\
\text { CareFusion AirLife }^{\text {TM }} \\
\text { nasal cannulae }\end{array}$ & Cut nasal cannulae \\
\hline \multirow[t]{2}{*}{ Improvised WHO } & $\begin{array}{l}\text { Standard low-flow wall oxygen } \\
\text { flow-meter (1-10lpm) }\end{array}$ & F\&P bubble CPAP chamber & F\&P bCPAP tubing & $\begin{array}{l}\text { High flow nasal cannula (West } \\
\text { Med) }\end{array}$ \\
\hline & $\begin{array}{l}\text { Standard low-flow wall oxygen } \\
\text { flow-meter (1-10lpm) }\end{array}$ & F\&P bubble CPAP chamber & Pumani tubing & Pumani Nasal Prongs \\
\hline
\end{tabular}



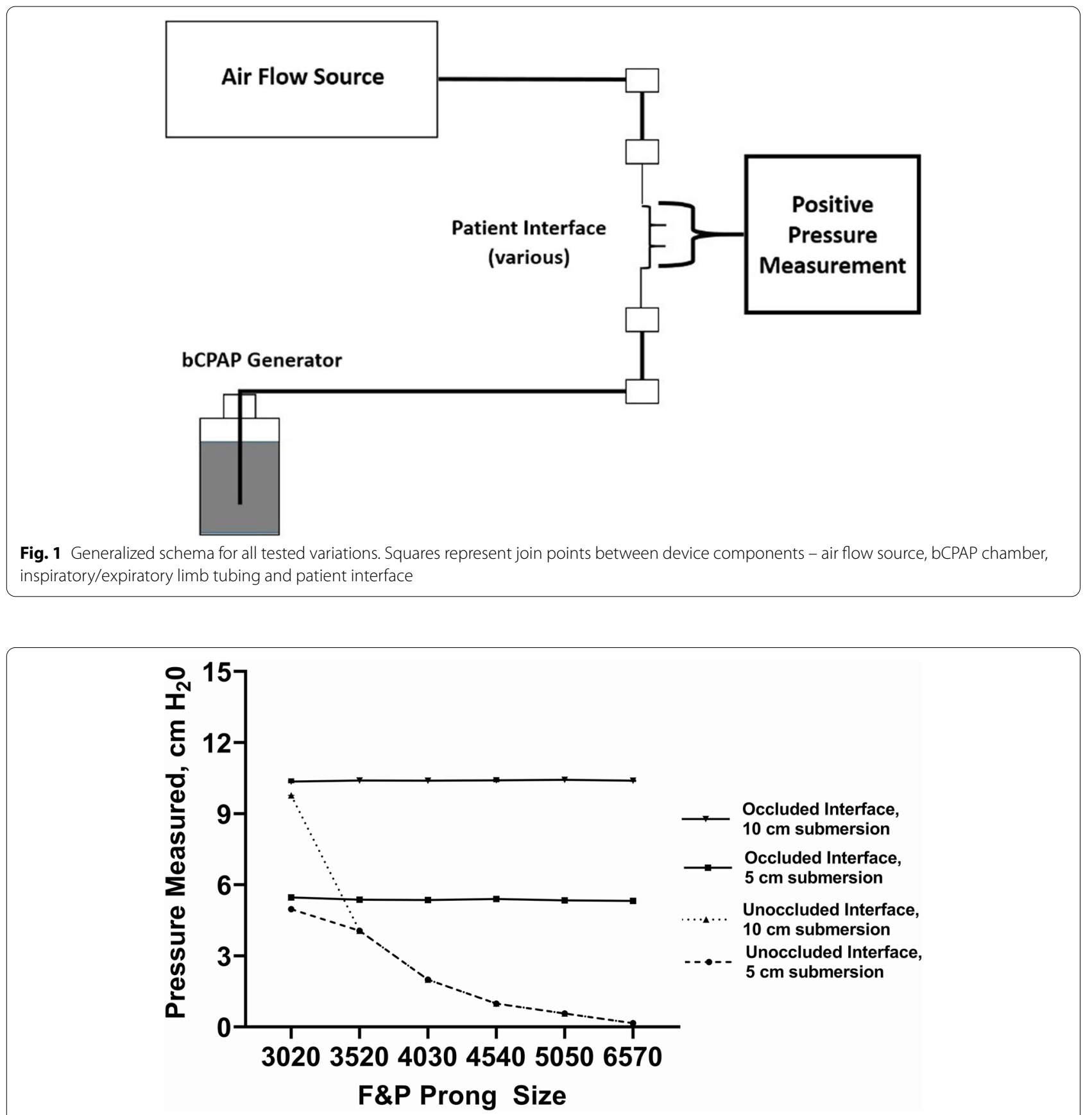

Fig. 2 F\&P bCPAP device with a F\&P circuit connected to F\&P Nasal Prongs - Measured Pressure [mean (SD), $\left.\mathrm{cm} \mathrm{H}_{2} \mathrm{O}\right]$. Testing performed at 8 Ipm with two submersion depths $-5 \mathrm{~cm} \mathrm{H}_{2} \mathrm{O}$ and $10 \mathrm{~cm} \mathrm{H}_{2} \mathrm{O}$. X-axis is categorical and not scaled to prong dimensions

low flow wall oxygen source $(1-10 \mathrm{lpm})$ with standard adult and pediatric size nasal cannulae (CareFusion Air$\left.\mathrm{Life}^{\mathrm{TM}}\right)$. The distal cut end of the nasal cannulae was submerged to a depth of 5 or $10 \mathrm{~cm}$ in the F\&P bubble CPAP chamber for testing (Fig. 5).

For improvised modifications to the WHO-style device (\#2), we tested the following modifications to the inspiratory and expiratory limbs as well as to the patient interface: (\#2a) substituting the Pumani respiratory circuit (tubing with a larger diameter than that recommended in the WHO-style device) connected to Pumani small and large nasal prongs in lieu of a standard lowflow nasal cannula (Fig. 6) and (\#2b) substituting the F\&P respiratory circuit (tubing with a larger diameter than 


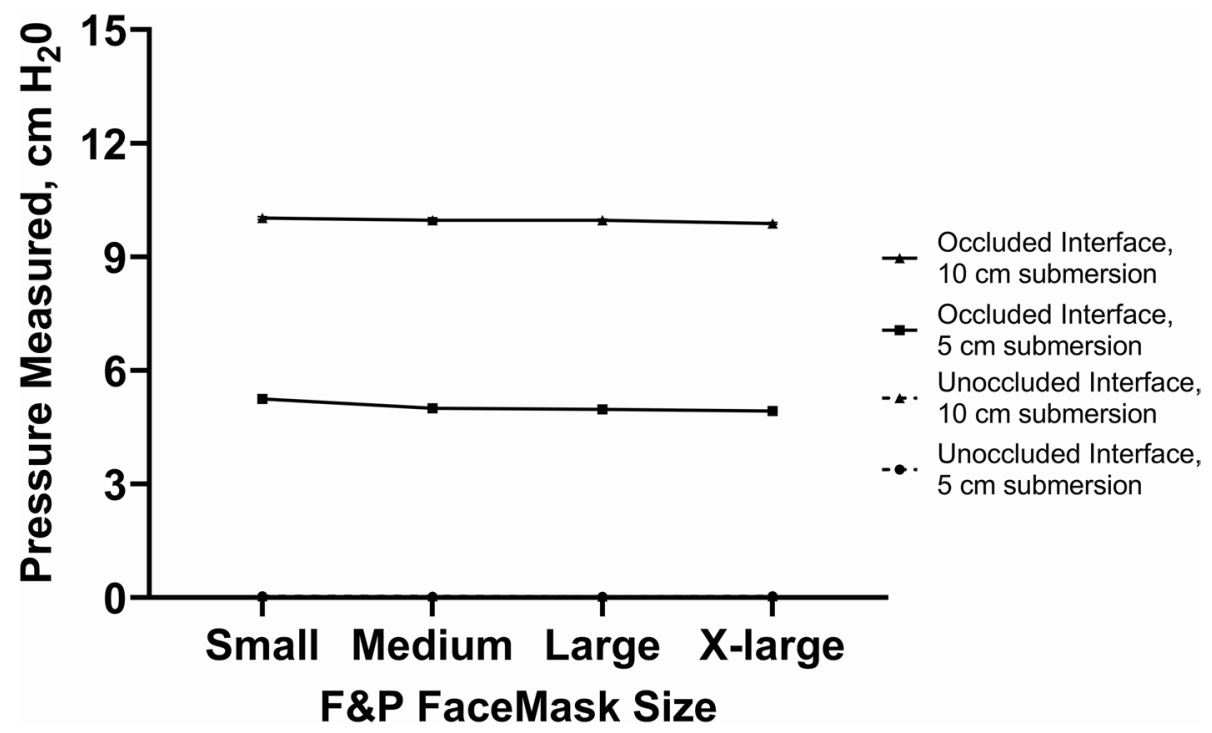

Fig. 3 F\&P bCPAP with a F\&P circuit connected to F\&P Nasal Masks - Measured Pressure [mean (SD), $\mathrm{cm} \mathrm{H}_{2} 0$ ]. Testing performed at $8 \mathrm{lpm}$ with two submersion depths $-5 \mathrm{~cm} \mathrm{H}_{2} \mathrm{O}$ and $10 \mathrm{~cm} \mathrm{H}_{2} \mathrm{O}$

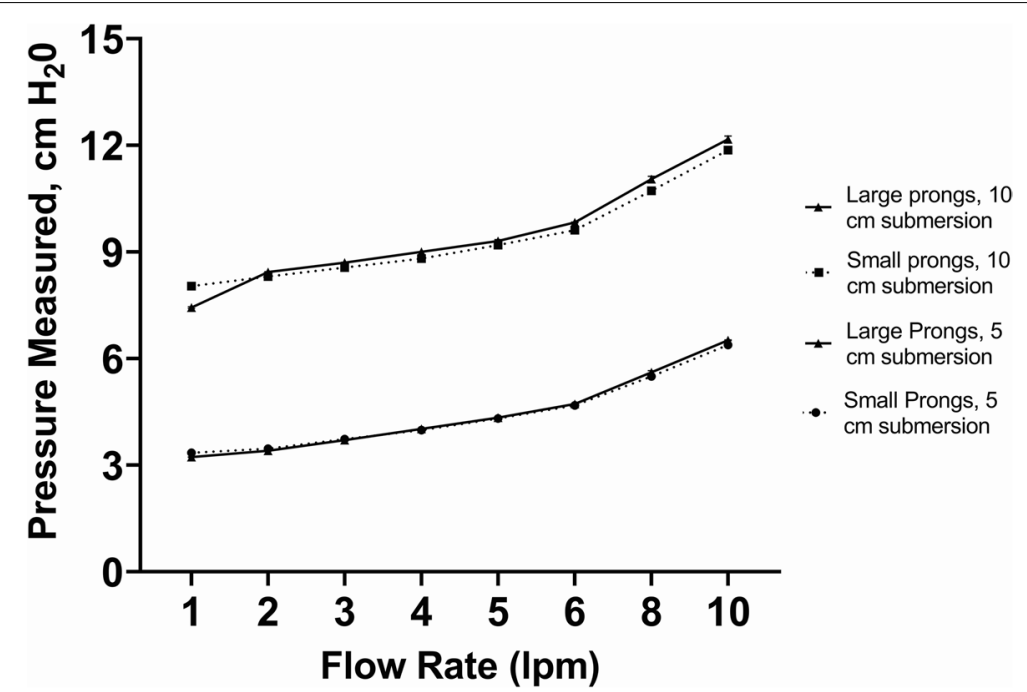

Fig. 4 Pumani bCPAP device with a Pumani circuit connected to small and large Pumani Nasal Prongs - Measured Pressure [mean (SD), $\left.\mathrm{cm} \mathrm{H}_{2} \mathrm{O}\right]$. Testing performed at two submersion depths $-5 \mathrm{~cm} \mathrm{H}_{2} \mathrm{O}$ and $10 \mathrm{~cm} \mathrm{H}_{2} \mathrm{O}$

that recommended in the WHO-style device) connected to a standard pediatric high flow nasal cannula (WestMed) in lieu of a standard low-flow nasal cannula over a range of flow rates (Fig. 7).

For the F\&P device with modifications (\#3), we tested the F\&P device substituting a standard Ambu-bag resuscitation mask attached to the end of the F\&P respiratory circuit in lieu of the F\&P nasal masks (Fig. 8).

For the Pumani device with modifications (\#4), we tested the Pumani device with Pumani circuit connected to F\&P nasal masks via an F\&P connector in lieu of Pumani nasal prongs (Fig. 9). We also tested the Pumani device using Pumani tubing connected to a typical commercially available CPAP/BIPAP mask used in older children (SleepWeaver ${ }^{\circledR}$, Circadiance, Export, PA, data not shown).

\section{Testing process}

The performance of all bCPAP devices was tested by measuring PP delivered at the patient interface using an American Healthcare Products Calibration Analyzer (Timeter 


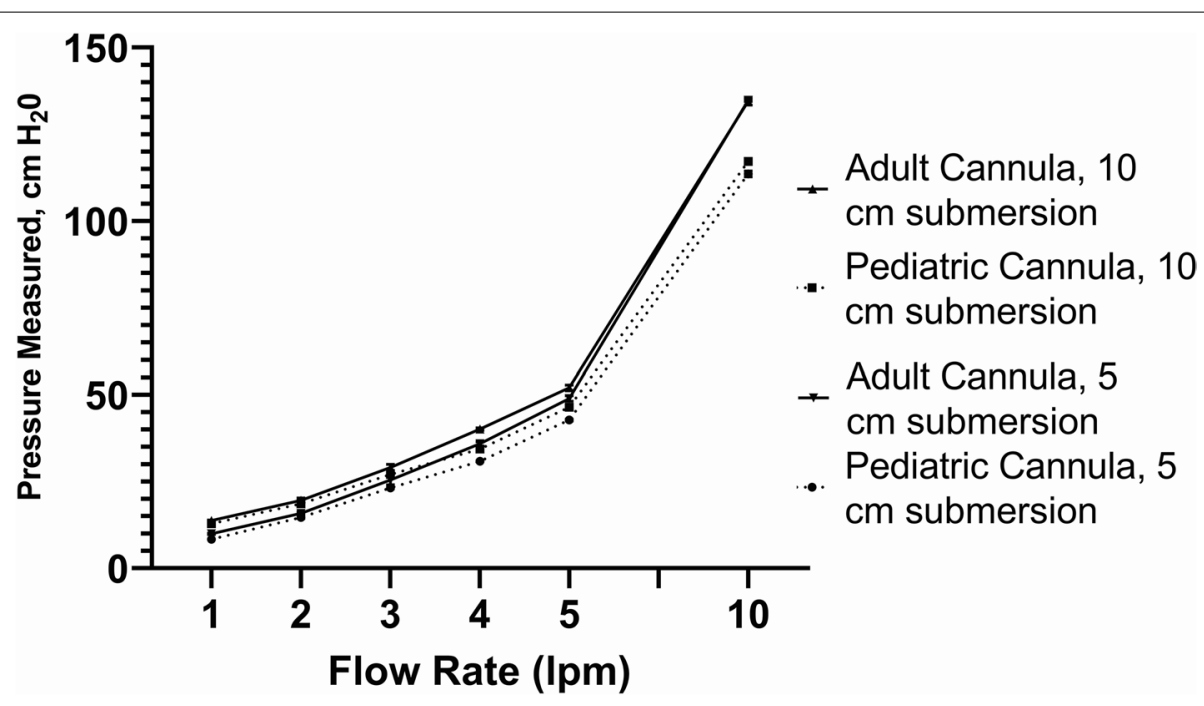

Fig. 5 WHO-style bCPAP device - Measured Pressure [mean (SD), $\mathrm{cm} \mathrm{H}_{2} \mathrm{O}$; note difference in scale]. Testing performed at two submersion depths $5 \mathrm{~cm} \mathrm{H}_{2} \mathrm{O}$ and $10 \mathrm{~cm} \mathrm{H}_{2} \mathrm{O}$

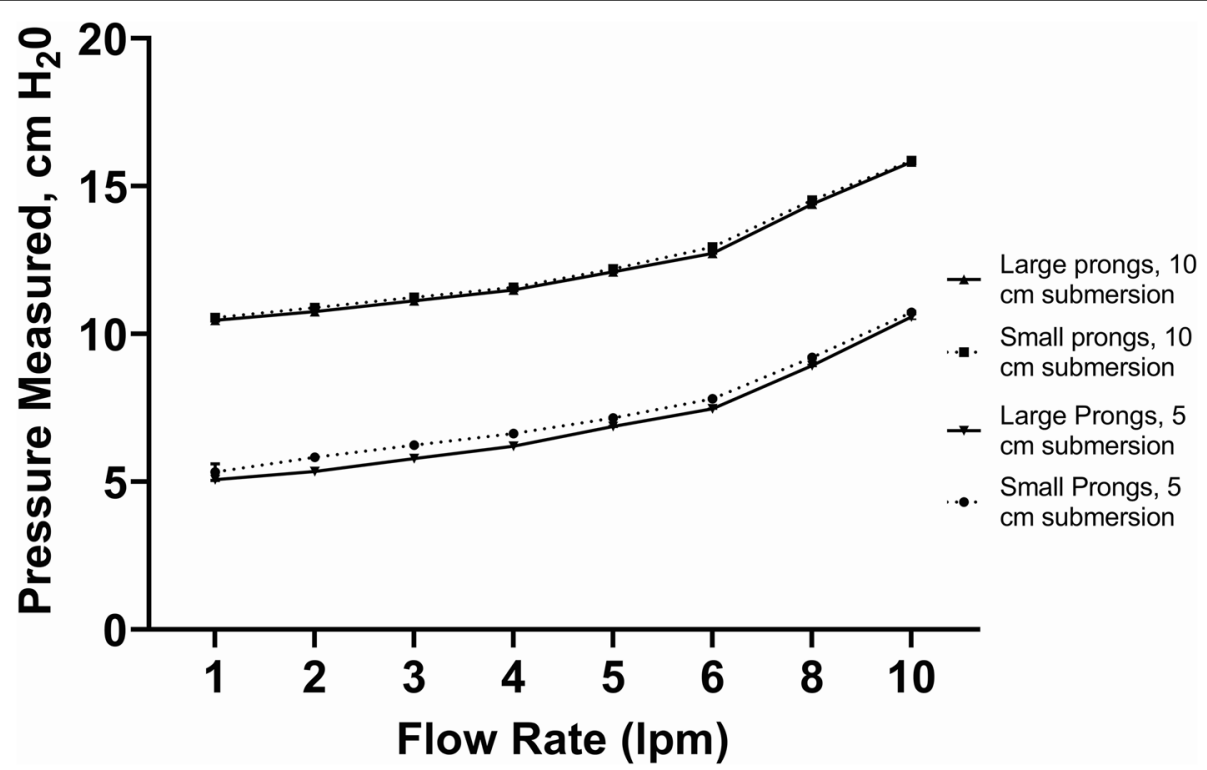

Fig. 6 WHO-style bCPAP device with a Pumani circuit connected to small and large Pumani Nasal Prongs - Measured Pressure [mean (SD), $\left.\mathrm{cm} \mathrm{H}_{2} \mathrm{O}\right]$. Testing performed at two submersion depths $-5 \mathrm{~cm} \mathrm{H}_{2} \mathrm{O}$ and $10 \mathrm{~cm} \mathrm{H}_{2} \mathrm{O}$

Instrument Corporation; series RT-200). Patient interfaces were connected directly to the measurement inlet probe of the analyzer without leakage to simulate $100 \%$ seal of the patient interface to the patient. Although we did not measure this formally, all device variations, tubing and patient interfaces were examined to ensure that the connections were tight and there was no obvious leakage. For the variations tested with the F\&P device, we were able to test positive pressure delivered under conditions where the prongs or masks were "fully occluded" vs. "fully unoccluded" to examine the range of possible PP generated as there are built-in access ports as part of the F\&P tubing. To test "fully occluded" scenarios we gently occluded the nasal prongs with our fingers. We occluded the nasal masks as well as resuscitation mask by pressing them gently on a flat smooth surface to ensure $100 \%$ seal. We acknowledge that this in vitro testing model is static testing and poorly replicates true bCPAP interactions with a live patient. However, 


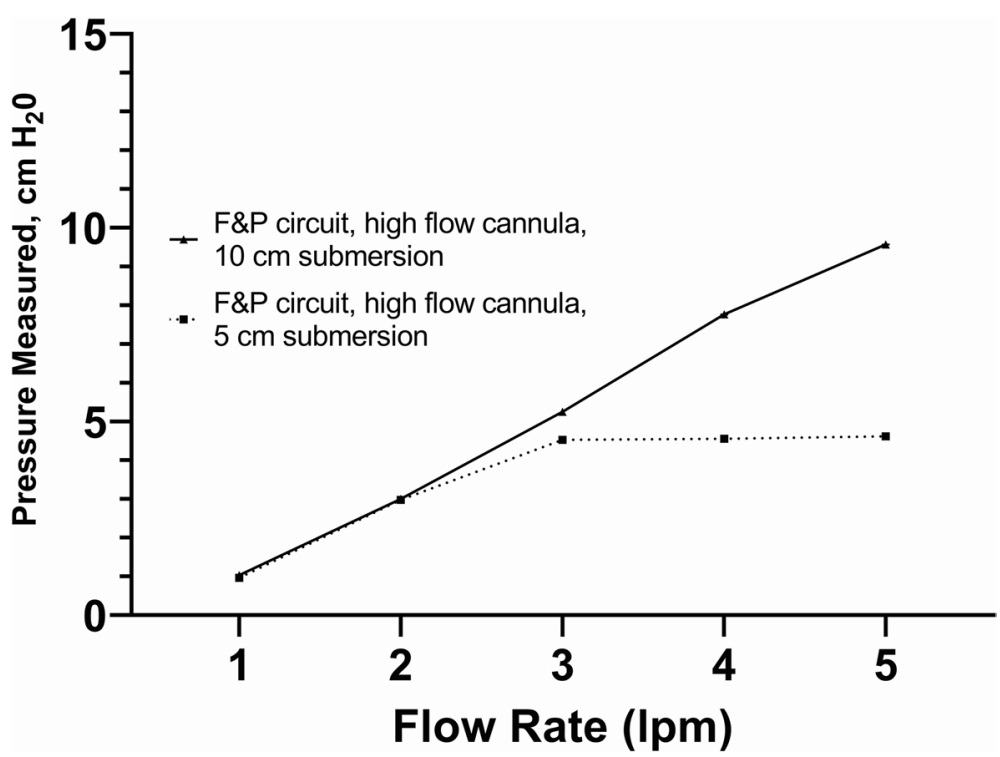

Fig. 7 WHO-style bCPAP device with an F\&P circuit connected to a standard high-flow nasal cannula - Measured Pressure [mean (SD), $\mathrm{cm} \mathrm{H}_{2} \mathrm{O} ;$, note testing only performed at flow rates $\leq 5 \mathrm{lpm}$ ]. Testing performed at two submersion depths $-5 \mathrm{~cm} \mathrm{H}_{2} \mathrm{O}$ and $10 \mathrm{~cm} \mathrm{H}_{2} \mathrm{O}$

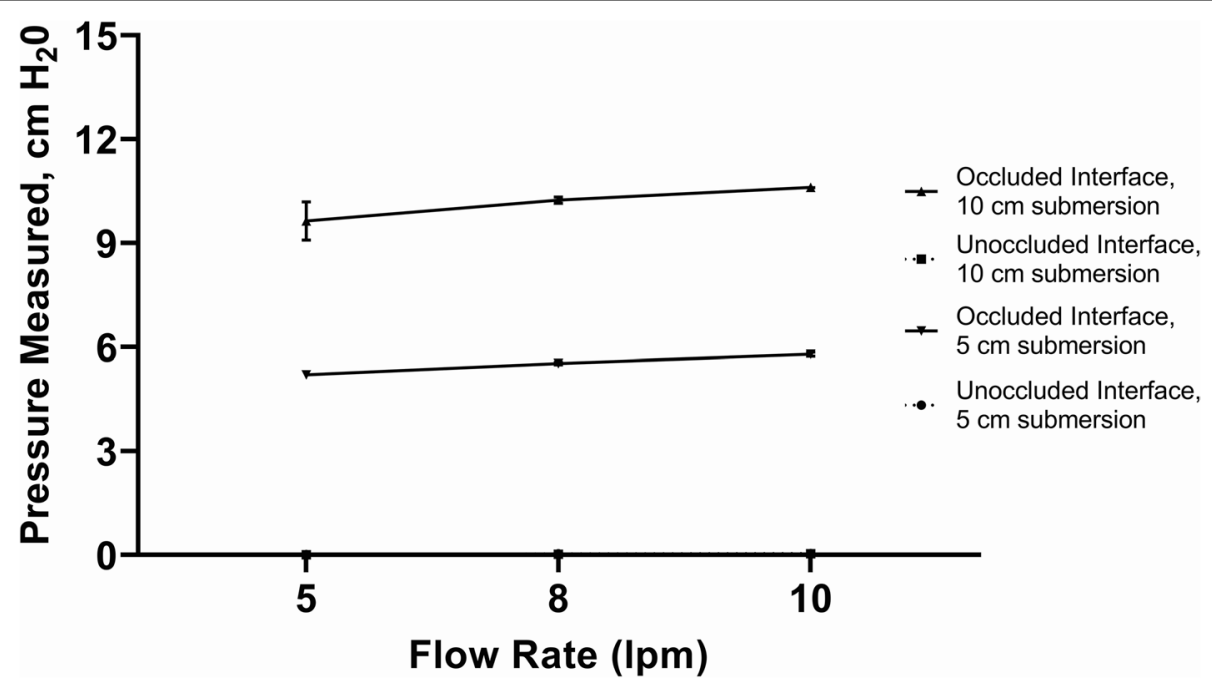

Fig. 8 F\&P bCPAP device with F\&P circuit connected to a standard Ambu-bag resuscitation face mask - Measured Pressure [mean (SD), $\left.\mathrm{cm} \mathrm{H}_{2} \mathrm{O}\right]$. Testing performed at two submersion depths $-5 \mathrm{~cm} \mathrm{H}_{2} \mathrm{O}$ and $10 \mathrm{~cm} \mathrm{H}_{2} \mathrm{O}$

the aim of our study was to measure the PP generated by several variations of bCPAP devices that our author group has observed used in real clinical practice in RLS.

\section{Flow rates}

Anecdotally, our author group has seen providers in RLS turn flow rates up intending to provide more support to patients. Typical oxygen sources in RLS include oxygen cylinders or oxygen concentrators. Cylinders deplete quickly if used with flow rates $>10 \mathrm{lpm}$ and oxygen concentrators most commonly generate $\leq 10 \mathrm{lpm}$. Therefore, to simulate this observation, we varied the flow rates between 1 and $10 \mathrm{lpm}$.

\section{Submersion depth}

Testing for all variations was performed at two submersion depths $-5 \mathrm{~cm} \mathrm{H}_{2} \mathrm{O}$ and $10 \mathrm{~cm} \mathrm{H}_{2} \mathrm{O}$ in the bubble CPAP chamber (F\&P or Pumani, see Table 1). 


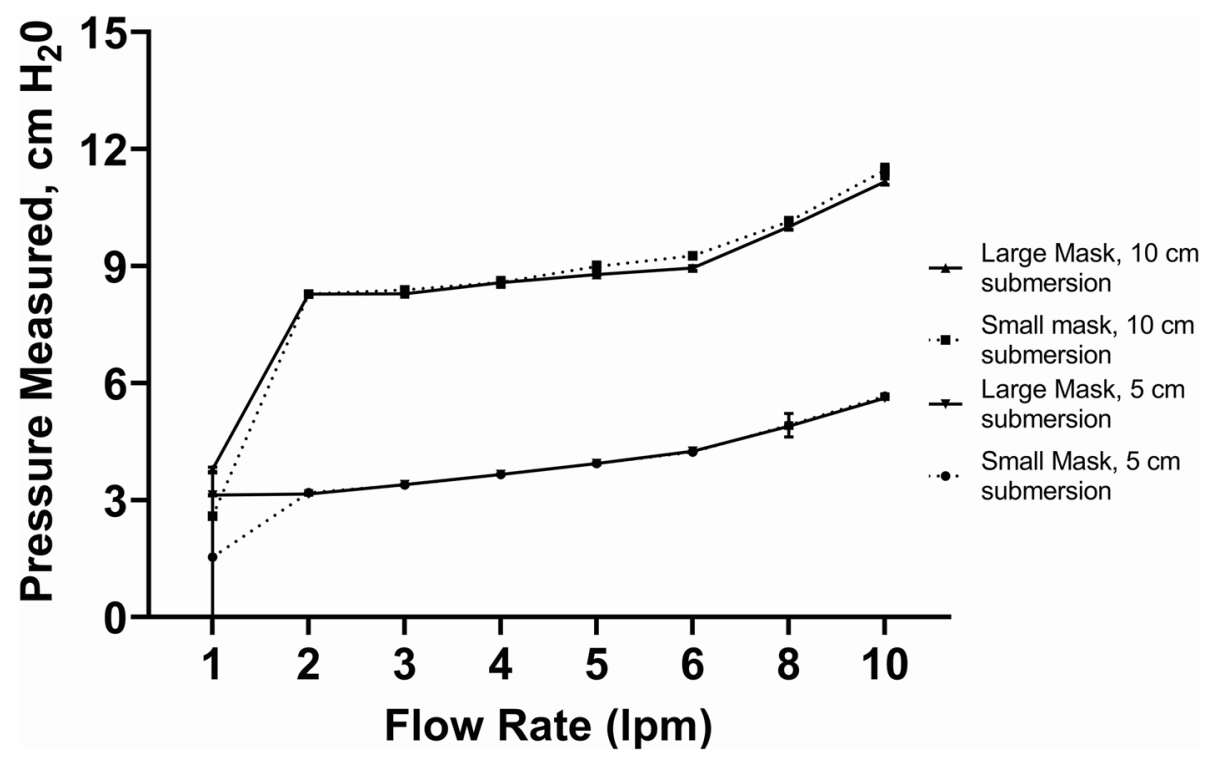

Fig. 9 Pumani bCPAP device with a Pumani circuit connected to small and large F\&P Nasal Masks - Measured Pressure [mean (SD), $\left.\mathrm{cm} \mathrm{H}_{2} \mathrm{O}\right]$. Testing performed at two submersion depths $-5 \mathrm{~cm} \mathrm{H}_{2} \mathrm{O}$ and $10 \mathrm{~cm} \mathrm{H}_{2} \mathrm{O}$

\section{Analysis}

Each condition was tested three times and the PP delivered was recorded. The analyzer was zeroed to room air between each measurement. Mean and standard deviation data were graphed using Prism v9.0 (GraphPad Software).

\section{Results}

To test our hypothesis, we compared PP delivered at the patient interface by the two commercially available bCPAP devices (Table 1, Figs. 2, 3 and 4) to PP delivered at the patient interface from the WHO-style, "low-tech" device and several improvised variations (Table 1, Figs. 5, $6,7,8$ and 9).

\section{Control devices}

Testing the F\&P device as recommend by the manufacturer with a range of $F \& P$ nasal CPAP prongs or with a range of $F \& P$ nasal masks, the delivered pressure matched the pressure set by the submersion depth in the bubble CPAP chamber and there was active, visible bubbling in the CPAP chamber (Figs. 2 and 3; 100\% occlusion; recommended flow rate of $8 \mathrm{lpm}$ flow). Of note, with larger nasal CPAP prong sizes and with all the nasal mask sizes, there was only visible bubbling when the prongs or masks were occluded. However, with the smallest F\&P nasal prongs (size 3020) there was visible bubbling in the CPAP chamber even with no occlusion of the prongs (Fig. 2).
For the Pumani device, we observed that with $100 \%$ occlusion of the prongs, there was bubbling in the chamber and measured pressures approximated or slightly exceeded (at higher flow rates) the submersion depth (Figs. 4 and $5 \mathrm{~cm}$ and $10 \mathrm{~cm} \mathrm{H}_{2} 0$ ). With the prongs unoccluded, there was no bubbling at all tested flow rates. There was no difference in measured pressures between the smallest and largest Pumani nasal prongs (sizes 0 [small], 5[large]) at either submersion depth or over a range of flow rates $(1-10 \mathrm{lpm}$, manufacturer's recommend flow rate is $6-8 \mathrm{lpm})$.

\section{Test devices}

For the WHO-style bCPAP devices (\#1), the measured pressures at the end of the nasal cannulae rapidly exceeded the submersion depths in the F\&P bubble CPAP-chamber even at low flow rates (Fig. 5) using both pediatric and adult nasal cannulae attached to a standard low-flow wall oxygen source at $1-10 \mathrm{lpm}$. For the improvised WHO-style device using a low flow wall-oxygen source with the Pumani respiratory circuit and Pumani nasal prongs (\#2a), the measured pressures approximated the submersion depths at lower flow rates, but began to exceed the submersion depths at higher flow rates (Fig. 6) for both small and large nasal prongs. For the variation utilizing F\&P tubing connected to a standard high flow nasal cannula (\#2b), the measured PP was less than the set submersion depths at flow rates $<5 \mathrm{lpm}$ (Fig. 7 ). 
For the improvised variation of the F\&P device with the F\&P circuit connected to an Ambu-bag resuscitation mask as the patient interface (\#3), the measured pressure matched the set depth in the CPAP chamber over a range of flow rates $(5-10 \mathrm{lpm})$, if there was $100 \%$ occlusion of the face mask (Fig. 8).

For the improvised variation of the Pumani device (\#4), using F\&P nasal masks as the patient interface, the measured pressures approximated the submersion depth in the Pumani bubble-chamber at all flow rates except at $1 \mathrm{lpm}$ (Fig. 9). The Pumani device combined with SleepWeaver ${ }^{\circledR}$ nasal mask was unable to generate any measurable PP at any flow rate (data not shown).

\section{Discussion}

Evidence to support the use of bCPAP for older infants and children in RLS is limited, has variable results and is of lower quality $[7,8]$. Based on our informal observations of the use of various WHO-style bCPAP devices used outside of the neonatal age range, we were concerned that the support provided for those older patients (i) may not achieve any PP benefiting the patient and thus may be wasting oxygen supply resources or (ii) may delay transfer to a higher level of care during a trial of therapy or (iii) may potentially be providing excessive PP which may be harmful to the patient.

Our results demonstrate that for the commercial bCPAP devices using flow rates recommended by the manufacturers, both F\&P and Pumani devices delivered at least the expected PP at the patient interface matching the submerged depth in the bubble-chamber, although the Pumani device had a steeper increase in resulting pressures (Figs. 2, 3 and 4). The etiology behind this performance difference is unclear although could be related to the performance of the flow pump inside the Pumani device at higher flow rates or the higher expiratory tubing resistance in the Pumani device [13]. It is important to note that with the F\&P device using the smallest size nasal prongs (size 3020), we observed bubbling in the bubble-chamber without occlusion, likely secondary to the intrinsic high resistance of this prong size [14]. The intrinsic resistance creates the potential for a provider to misjudge the level of PP support actually being provided to the child, regardless of the actual leakage at the patient interface [15]. Chamber bubbling was a reliable proxy for occlusion with the Pumani device, with the F\&P nasal masks and with the resuscitation face mask (Figs. 3, 4 and 8). However, we strongly recommend against using the resuscitation face mask in this manner because of significant safety concerns related to possible hypercarbia, risk for aspiration or gastrointestinal perforations $[7,16]$.

With the standard WHO-style bCPAP device, the cut nasal cannulae generated PP that far exceeded the set depth in the F\&P bCPAP chamber (Fig. 5). The data from the pediatric cannula were uniformly lower than the data from the adult cannula, suggesting the possibility of unmeasured leakage. These high measured pressures were also observed in a recent publication comparing narrow and wide-diameter bCPAP circuits [12]. The high PP occurred only with $100 \%$ occlusion of the nasal cannulae, but these data raise concern that in small infants, especially with higher flow rates, the pressure delivered could create discomfort, intolerance or barotrauma in an infant leading to use of resources without benefit. Testing a variation of the standard WHO-style bCPAP device with larger diameter F\&P tubing (a combination observed in RLS by our group) prevented the generation of excessive PP values (Fig. 7). However, this improvised device was not able to generate appropriate pressures at lower flow rates, likely secondary to leaks in the system, although we did not formally test for this possibility. However, this was still a concerning result as some providers may think that a low flow rate could be a beneficial strategy to stretch limited oxygen resources while still providing PP. Our results demonstrate that using low flow rates for this variation provides oxygen only with little to no PP benefit. An improvised variation employing the Pumani device connected to F\&P nasal masks also generated appropriate levels of PP, except at very low liter flow rates (Fig. 9), as did utilizing a low-flow oxygen source connected to the Pumani tubing and prongs (Fig. 6). Interestingly, the combination of low flow oxygen source, F\&P bubble chamber and Pumani tubing/interface differed from the complete Pumani data at high flow rates (Fig. 4). The differences observed between Fig. 4 and Fig. 6 and the small standard deviation measurements could be due to unmeasured leakage or differences in the actual air flow delivered by the Pumani air pump versus a low flow wall oxygen source.

F\&P nasal prongs are designed to "fill the nares completely without stretching the skin" (F\&P bCPAP brochure). This is to create a seal without causing tissue pressure injuries. In our testing, PP was only consistently achieved when there was $100 \%$ occlusion of the distal end of the apparatus (prongs or mask). If the apparatus was not occluded (as would be the case in older children), the PP delivered was necessarily less than the submersion depth. This observation has particular relevance when considering the implications of attempting to provide PP to older children in clinical contexts such as septic shock. When treating septic shock, PP (non-invasive or invasive) is often administered with a goal to not only reduce work of breathing, but also to reduce left ventricular afterload (assuming adequate preload), thereby reducing the overall metabolic consumption of oxygen which can help to optimize oxygen delivery [17]. In shock 
states, failure to achieve these goals can have detrimental, potentially fatal, effects. This physiology underscores the risk of using improvised low-tech bCPAP devices that are used outside of previously tested age-ranges.

Either soft cloth or gel-plastic nasal masks are the patient interface typically used to deliver CPAP or BIPAP to older children in resource-replete settings. Testing the Pumani device with a typical commercially available CPAP/BIPAP mask designed for older children (SleepWeaver $^{\mathrm{TM}}$, Circadiance, Export, PA) did not generate any measurable PP (Table 1, data not shown) due to the inherent controlled leakage of the mask, which the low liter bias flow rate of Pumani was not able to overcome. Typical commercially available CPAP/BIPAP masks designed for older children have vented interfaces and require high liter bias flow rates of $>40 \mathrm{lpm}$ to function appropriately and prevent $\mathrm{CO}_{2}$ rebreathing [18]. Importantly, in a RLS, flow rates greater than $10 \mathrm{lpm}$ are practically hard to generate. Oxygen cylinders deplete quickly if used at $>10 \mathrm{lpm}$, raising stability of supply chain issues if the liter flow rate is raised $>10 \mathrm{lpm}$. Typical electric oxygen concentrators only generate $\leq 10 \mathrm{lpm}$. Therefore, it may not be possible to provide CPAP/BIPAP to older children whose faces/noses are too large to fit a nonvented nasal mask (e.g. an F\&P device) in the absence of reliable, built-in wall-piped-high-flow oxygen or air (rare in a RLS) or without a device employing a self-contained flow pump.

There are several limitations to this in vitro study. The devices and improvisations were tested in static conditions, in the absence of any type of lung simulator and were not attached to any type of simulated pharynx, preventing the estimation of pharyngeal pressures and any step down in pressure transmission [19]. With static testing, the choice of interface should not significantly affect the generated pressure unless the outlet resistance is markedly higher. This phenomenon was only observed with the smallest F\&P prong set similar to prior observations, [14] where the authors observed a large increase in resistance for the F\&P 3020 prongs independent of flow rate. We did test the F\&P device with fully occluded and fully-unoccluded conditions, but this does not appropriately simulate the effect of having the mouth open or closed. We only tested flows $\leq 10 \mathrm{lpm}$ and did not measure air flow leakage for any device. Reduction of leakage is crucial for PP generation [15].

Although there have been some attempts to create higher flow devices with Venturi valves that can service older patients (e.g. ECPAP), [20] we are hopeful that the emergence of high flow nasal cannula devices which have built-in flow pumps and which can generate flows up to $60 \mathrm{lpm}$ without a high-pressure wall air source (e.g. F\&P
Airvo $^{\mathrm{TM}} 2$ system), as well as the general robustness of the Pumani model, point to the possible future development of new innovative devices that could safely, successfully and reliably generate non-invasive positive pressure without high-pressure wall-piped air/oxygen in a RLS for older children.

\section{Conclusion}

In well-resourced settings, a trial of non-invasive respiratory support to avoid endotracheal intubation and mechanical ventilation is generally accepted as safe and effective therapy for infants and children with moderate to severe respiratory illnesses. All of these interventions require high-level technical knowledge and robust supply chains which may be scarce in RLS [21]. Bubble CPAP has been proposed as an alternative in resource-limited settings to meet the need for respiratory support for infants [6]. However, there is an urgent need to develop reliable non-invasive positive pressure capacity for children above the infant age range.

All of the WHO-style bCPAP devices that we tested were able to generate PP that matched, or markedly exceeded, the submersion depth set in the associated bubble-chamber. PP was only measurable with $100 \%$ seal of the distal end of the bCPAP patient interfaces (commercial or improvised) but perfect seal is anatomically unlikely in older children. Therefore, the use of WHOstyle bCPAP devices in patients outside the infant age range may be limited. Our data do support the effectiveness of bCPAP to generate appropriate pressures in newborns and young infants. However, based on our results, we recommend against using WHO-style bCPAP devices for non-infant patients with respiratory failure due to the low likelihood of any PP generation and instead recommend using standard oxygen therapy with nasal cannulae or face-masks, as well as early consideration of transfer to a higher level of care.

\section{Abbreviations \\ RLS: Resource limited setting; CPAP: Continuous positive airway pressure; bCPAP: Bubble CPAP; Ipm: Liters per minute; F\&P: Fisher \& Paykel; BIPAP: Bilevel positive airway pressure; PP: Positive pressure. \\ Acknowledgments \\ The authors would like to thank Megan Heenan, Ph. D, Director of Technol- ogy Development, Rice $360^{\circ}$ Institute of Global Health for assistance in obtaining a Pumani device and for critical comments and suggestions for this study.}

\section{Authors' contributions}

$N E$, NS, JW conceptualized and designed the study, carried out the initial analyses, collected data and reviewed and revised the manuscript. ST, MH conceptualized and designed the study and reviewed and revised the manuscript. RN provided materials, conceptualized and designed the study, collected data and reviewed and revised the manuscript. All authors read and approved the final manuscript. 


\section{Funding}

There was no funding for this project.

\section{Availability of data and materials}

All data generated or analyzed during this study are included in this published article as graphs and all raw data are available from the corresponding author on reasonable request.

\section{Declarations}

\section{Ethics approval and consent to participate}

Not applicable.

\section{Consent for publication}

Not applicable.

\section{Competing interests}

The authors declare that they have no competing interests.

\section{Author details}

'Division of Critical Care, Department of Pediatrics, Baylor College of Medicine/ Texas Children's Hospital, 6651 Main Street, MC: E1420, Houston, TX 77030, USA. ${ }^{2}$ Department of Respiratory Therapy, Texas Children's Hospital, Houston, TX, USA. ${ }^{3}$ Division of Hospital Medicine, Department of Pediatrics, Baylor College of Medicine/Texas Children's Hospital, Houston, TX, USA. ${ }^{4}$ Senior Global RMNCH Clinical and Technical Advisor, Rice $360^{\circ}$ Institute for Global Health, Houston, TX, USA. ${ }^{5}$ Global Child Health Group, Emma Children's Hospital, Amsterdam University Medical Center, Amsterdam, the Netherlands. ${ }^{6}$ Princess Máxima Center for Pediatric Oncology, Utrecht, the Netherlands. ${ }^{7}$ Division of Emergency Medicine, Department of Pediatrics, Baylor College of Medicine/ Texas Children's Hospital, Houston, TX, USA.

\section{Received: 25 May 2021 Accepted: 9 November 2021}

\section{Published online: 27 November 2021}

\section{References}

1. Pneumonia Etiology Research for Child Health Study Group. Causes of severe pneumonia requiring hospital admission in children without HIV infection from Africa and Asia: the PERCH multi-country case-control study. Lancet. 2019;394(10200):757-79.

2. Muttalib F, Gonzalez-Dambrauskas S, Lee JH, Steere M, Agulnik A, Murthy $S$, et al. Pediatric emergency and critical care resources and infrastructure in resource-limited settings: a multicountry survey. Crit Care Med. 2021:49(4):671-81.

3. Mwatha AB, Mahande M, Olomi R, John B, Philemon R. Treatment outcomes of Pumani bubble-CPAP versus oxygen therapy among preterm babies presenting with respiratory distress at a tertiary hospital in Tanzania-randomised trial. PLoS One. 2020;15(6):e0235031.

4. Brown J, Machen $\mathrm{H}$, Kawaza K, Mwanza Z, Iniguez S, Lang H, et al. A highvalue, low-cost bubble continuous positive airway pressure system for low-resource settings: technical assessment and initial case reports. PLoS One. 2013;8(1):e53622.

5. Kawaza K, Machen HE, Brown J, Mwanza Z, Iniguez S, Gest A, et al. Efficacy of a low-cost bubble CPAP system in treatment of respiratory distress in a neonatal ward in Malawi. PLoS One. 2014;9(1):e86327.
6. Won A, Suarez-Rebling D, Baker AL, Burke TF, Nelson BD. Bubble CPAP devices for infants and children in resource-limited settings: review of the literature. Paediatr Int Child Health. 2019;39(3):168-76.

7. McCollum ED, Mvalo T, Eckerle M, Smith AG, Kondowe D, Makonokaya $D$, et al. Bubble continuous positive airway pressure for children with high-risk conditions and severe pneumonia in Malawi: an open label, randomised, controlled trial. Lancet Respir Med. 2019;7(11):964-74.

8. Chisti MJ, Salam MA, Smith JH, Ahmed T, Pietroni MA, Shahunja KM, et al. Bubble continuous positive airway pressure for children with severe pneumonia and hypoxaemia in Bangladesh: an open, randomised controlled trial. Lancet. 2015;386(9998):1057-65.

9. Duke T. CPAP: a guide for clinicians in developing countries. Paediatr Int Child Health. 2014;34(1):3-11.

10. World Health O. Oxygen therapy for children: a manual for health workers. Geneva: World Health Organization; 2016. p. 2016.

11. Midwest Consortium of Global Child Health E. SUGAR PEARLS: Midwest Consortium of Global Child Health Educators; [Available from: https:// sugarprep.org/videos/.

12. Baldursdottir S, Falk M, Donaldsson S, Jonsson B, Drevhammar T. Basic principles of neonatal bubble CPAP: effects on CPAP delivery and imposed work of breathing when altering the original design. Arch Dis Child Fetal Neonatal Ed. 2020;105(5):550-4.

13. Heenan M, Rojas JD, Oden ZM, Richards-Kortum R. In vitro comparison of performance including imposed work of breathing of CPAP systems used in low-resource settings. PLoS One. 2020;15(12):e0242590.

14. Green EA, Dawson JA, Davis PG, De Paoli AG, Roberts CT. Assessment of resistance of nasal continuous positive airway pressure interfaces. Arch Dis Child Fetal Neonatal Ed. 2019;104(5):F535-f9.

15. Falk M, Gunnarsdottir K, Baldursdottir S, Donaldsson S, Jonsson B, Drevhammar T. Interface leakage during neonatal CPAP treatment: a randomised, cross-over trial. In: Archives of disease in childhood Fetal and neonatal edition; 2021.

16. Garland JS, Nelson DB, Rice T, Neu J. Increased risk of gastrointestinal perforations in neonates mechanically ventilated with either face mask or nasal prongs. Pediatrics. 1985;76(3):406-10.

17. Bronicki RA, Anas NG. Cardiopulmonary interaction. Pediatr Crit Care Med. 2009;10(3):313-22.

18. Medrinal C, Prieur G, Contal O, Villiot-Danger E, Doyle C, Reychler G, et al. Non-invasive ventilation: evaluation of $\mathrm{CO} 2$ washout by intentional leaking in three recent oronasal masks. A pilot study. Minerva Anestesiol. 2015;81(5):526-32.

19. De Paoli AG, Lau R, Davis PG, Morley CJ. Pharyngeal pressure in preterm infants receiving nasal continuous positive airway pressure. Arch Dis Child Fetal Neonatal Ed. 2005;90(1):F79-81.

20. Pons-Òdena M, Valls A, Grifols J, Farré R, Cambra Lasosa FJ, Rubin BK. COVID-19 and respiratory support devices. Paediatr Respir Rev. 2020;35:61-3.

21. Parker MM, Nuthall G, Brown C 3rd, Biagas K, Napolitano N, Polikoff LA, et al. Relationship between adverse tracheal intubation associated events and PICU outcomes. Pediatr Crit Care Med. 2017;18(4):310-8.

\section{Publisher's Note}

Springer Nature remains neutral with regard to jurisdictional claims in published maps and institutional affiliations. 